\title{
A PARTIAL BAER *-SEMIGROUP OF RELATIONS
}

\author{
R. H. SCHELP \\ (Received 18 August 1971) \\ Communicated by P. D. Finch
}

\section{Introduction}

It is shown in Gudder and Schelp (1970) that partial Baer*-semigroups coordinatize orthomodular partially ordered sets (orthomodular posets). This means for $P$ an orthomodular poset there exists a partial Baer $*$-semigroup whose closed projections are order isomorphic to $P$ preserving ortho-complementation. This coordinatization theorem generalizes Foulis (1960) in which orthomodular lattices are coordinatized by Baer *-semigroups. In particular Foulis (unpublished) shows that any complete atomic Boolean lattice is coordinatized by a Baer *-semigroup of relations. Since Greechie (1968), (1971) shows that a whole class of orthomodular posets can be formed by "pasting" together Boolean lattices, it is natural to consider the following problem. Let $\mathscr{S}$ be a family of Baer *-semigroups of relations which coordinatize the family $\mathscr{B}$ of complete atomic lattices. Is it possible to construct a partial *-semigroup of relations $\mathscr{R}$ which contains each member of $\mathscr{P}$ such that when $P$ is an orthomodular poset obtained by a "Greechie pasting" of members of $\mathscr{B}$ then $\mathscr{R}$ coordinatizes $P$ ? This question is considered in the sequel and answered affirmatively for a certain subclass of "Greechie pasted" orthomodular posets. In addition the construction of $\mathscr{R}$ nicely fulfills another objective in that it provides us with "nontrivial" coordinate partial Baer *-semigroups for a whole family of well known orthomodular posets. This is particularly significant since the only other known coordinate partial Baer *-semigroups, for those posets in this family which are not lattices, are the "minimal" ones given in Gudderand and Schelp (1970).

\section{Construction of the partial Baer *-semigroup}

We first review the definition of a partial Baer *-semigroup as given in Gudder and Schelp (1970). Let $S$ be a set, $R \subseteq S \times S$ a relation, and $(x, y) \rightarrow x y$ a map from $R$ into $S$. The pair $(S, R)$ is a partial semigroup if for $x, y, z \in S$ with $(x, y),(y, z) \in R$ we have $(x y, z) \in R$ if and only if $(x, y z) \in R$ and in that case $(x y) z=x(y z)$. If 
$R)(S$, hasa zero 0 (i.e., $(0, x),(x, 0) \in R$ for every $x \in S$ with $0 x=x 0=0)$ and in addition there exist there exist maps ' and * from $S$ into $S$ such that

(a) $x=x^{* *}$ for all $x \in S$,

(b) $(x, y) \in R$ implies $\left(y^{*}, x^{*}\right) \in R$ with $(x y)^{*}=y^{*} x^{*}$,

(c) $\left(x^{\prime}, x^{\prime}\right) \in R$ and $x^{\prime} x^{\prime}=x^{*}=x^{\prime}$ for all $x \in S$, i.e., $x^{\prime}$ is a projection, and

(d) $(x, y) \in R$ with $x y=0$ if and only if $\left(x^{\prime}, y\right) \in R$ with $y=x^{\prime} y$,

then the quadruple $\left(S, R,{ }^{*},{ }^{\prime}\right)$ is called a partial Baer *-semigroup. For conciseness we will frequently reference such quadruples by a single symbol $S$. The elements of the set $\left\{x^{\prime} \mid x \in S\right\}$ are called the closed projections of $S$ and this set is denoted by $P^{\prime}(S)$. The set $P^{\prime}(S)$ is ordered by defining $e \leqq f$ in $P^{\prime}(S)$ if an only if $(e, f) \in R$ and $e=e f$. Whenever $R=S \times S$ the partial Baer *-semigroup becomes the Baer *-semigroup defined in Foulis (1960). A partial semigroup satisfying conditions (a) and (b) alone is called a partial involution semigroup with ${ }^{*}$ as involution.

Before proceeding to the construction of the partial Baer *-semigroup of relations it is best to glimpse at the motivation behind it. Foulis (unpublished) considers the set of relations $\mathscr{P}(X \times X)$, the power set of $X \times X$, on a non-empty set $X$. For $S, T \in \mathscr{P}(X \times X)$ he defines the product $S T$ to be the composition of the relations $S$ and $T$, that is,

$$
S T=\{(x, y) \mid \text { there exists a } z \in X \text { such that }(x, z) \in S \text { and }(z, y) \in T\} .
$$

Also * and ' are defined on $\mathscr{P}(X \times X)$ by

$$
S^{*}=\{(y, x) \mid(x, y) \in S\} \text { and } S^{\prime}=\Delta_{\bar{X}} \cap(M \times M)
$$

where $S \in \mathscr{P}(X \times X), \Delta_{X}$ is the identity relation on $X$, and

$$
M=X \backslash\{y \in X \mid(x, y) \in S \text { for some } x \in X\} .
$$

$\mathscr{P}(X \times X)$ with $*$ and ' as just defined is a Baer $*$-semigroup. Furthermore $\mathscr{P}(X \times X)$ coordinatizes the Boolean lattice $(\mathscr{P}(X), \subseteq, c)$ where $c$ denotes set complementation, that is

$$
\left(P^{\prime}(\mathscr{P}(X \times X)), \leqq,^{\prime}\right)
$$

is isomorphic to $(\mathscr{P}(X), \subseteq c)$. Now Greechie (1965), (1971) shows that certain orthomodular posets can be constructed by properly "pasting" together Boolean lattices. Thus the objective is to generalize the Baer $*$-semigroup of relations $\mathscr{P}(X \times X)$ to a partial Baer *-semigroup of relations $\mathscr{R}$ on a family $\left\{X_{\alpha}\right\}_{\alpha \in \Gamma}$ of sets such that if $P$ is an orthomodular poset obtained by a "Greechie pasting" of the Boolean lattices $\left\{\mathscr{P}\left(X_{\alpha}\right)\right\}_{\alpha \in \Gamma}$, then $P$ is isomorphic to $P^{\prime}(\mathscr{R})$. Furthermore $\mathscr{R}$ should be constructed so that each $\mathscr{P}\left(X_{\alpha} \times X_{\alpha}\right)$ is contained in $\mathscr{R}$. The partial Baer *-semigroup we construct does fulfill each of these objectives for a certain subclass of "pasted" orthomodular posets. 
To facilitate the construction we first introduce much notation and several definitions. Unless otherwise specified, this notation will be used throughout the remainder of this paper. Let $\left\{X_{\alpha}\right\}_{\alpha \in \Gamma}$ be a collection of sets and $X=\bigcup_{\alpha \in \Gamma} X_{\alpha}$. $\Delta_{X}$ denotes the identity relation on $X \times X$. If $M \subseteq X_{\alpha}$, for some $\alpha \in \Gamma$, let

$$
\Gamma(M)=\left\{\beta \in \Gamma \mid M \subseteq X_{\beta}\right\},
$$

$|\Gamma(M)|$ denote the cardinality of $\Gamma(M)$,

and

$$
K(M)=\bigcup_{\beta \in \Gamma(M)}\left(X_{\beta} \mid M\right),
$$

Define

$$
M^{c}=\Delta_{X} \cup(K(M) \times K(M)) .
$$

$$
\begin{gathered}
\mathscr{R}_{1}(X)=\bigcup_{\alpha \beta \in \Gamma} \mathscr{P}\left(X_{\alpha} \times X_{\beta}\right), \\
\mathscr{R}_{2}(X)=\left\{M^{c} \mid M \subseteq X_{\alpha} \text { for some } \alpha \in \Gamma \text { and } M^{c} \notin \mathscr{R}_{1}(X)\right\},
\end{gathered}
$$

and $\mathscr{R}(X)=\mathscr{R}_{1}(X) \cup \mathscr{R}_{2}(X)$. For $S \in \mathscr{R}(X)$ let

$$
\operatorname{ran} S=\{y \in X \mid \text { there exists an } x \in X \text { such that }(x, y) \in S\}
$$

and

$$
\operatorname{dom} S=\{x \in X \mid \text { there exists a } y \in X \text { such that }(x, y) \in S\} .
$$

Define a relation $\rho$ on $\mathscr{R}(X)$ as follows:

$$
\begin{aligned}
& \rho=\bigcup_{i=1}^{4} \rho_{i} \text { where } \\
& \rho_{1}=\left\{(S, T) \mid S, T \in \mathscr{R}_{1}(X) \text { and there exists an } \alpha \in \Gamma \text { such that (ran } S\right) \text {, } \\
& \left.(\operatorname{dom} T) \subseteq X_{\alpha}\right\}, \\
& \rho_{2}=\left\{\left(M^{c}, N^{c}\right) \mid M^{c}, N^{c} \in \mathscr{R}_{2}(X) \text { with } M \subseteq N \text { or } N \subseteq M\right\} \text {, } \\
& \rho_{3}=\left\{\left(M^{c}, S\right) \mid S \in \mathscr{R}_{1}(X), M^{c} \in \mathscr{R}_{2}(X) \text {, and there exists an } \alpha \in \Gamma\right. \text { such that } \\
& \left.M,(\operatorname{dom} S) \subseteq X_{\alpha}\right\} \text {, and } \\
& \rho_{4}=\left\{\left(S, M^{c}\right) \mid S \in \mathscr{R}_{1}(X), M^{c} \in \mathscr{R}_{2}(X) \text {, and there exists an } \alpha \in \Gamma\right. \text { such that } \\
& \left.M,(\operatorname{ran} S) \subseteq X_{\alpha}\right\} \text {. }
\end{aligned}
$$

For $(S, T) \in \rho$ define $S T$ as the composition of the relations $S$ and $T$. Also for $S \in \mathscr{R}(X)$ define $S^{*}=\{(y, x) \mid(x, y) \in S\}$.

The construction depends upon the collection $\left\{X_{\alpha}\right\}_{\alpha \in \Gamma}$ satisfying certain conditions. We thus give a convention.

CONVENTION 2.1. Let $\left\{X_{\alpha}\right\}_{\alpha \in \Gamma}$ be a nonempty collection of sets such that $X_{\alpha} \neq X_{\beta}$ for $\alpha \neq \beta$ in $\Gamma$. Also let $X=\bigcup_{\alpha \in \Gamma} X_{\alpha}$. We assume that the collection satisfies the following two additional conditions. 
(AS) If $\alpha, \beta, \gamma \in \Gamma$ and $M, N, Q$ are subsets of $X$ such that $M \subseteq X_{\alpha} \cap X_{\beta}$, $N \subseteq X_{\alpha}, Q \subseteq X_{\beta}$, and $(N \backslash M), Q \subseteq X_{\gamma}$, then there exists a $\delta \in \Gamma$ such that $N,(Q \backslash M) \subseteq X_{\delta}$.

(UP) If there exist $\alpha, \beta \in \Gamma$ and sets $M \subseteq X_{\alpha}, N \subseteq X_{\beta}$ such that $K(M)$ $=K(M)$ with $K(M) \nsubseteq X_{\gamma}$ for all $\gamma \in \Gamma$, then $M=N$.

Several remarks concerning the above convention are in order. We will show that when the sets $\left\{X_{\alpha}\right\}_{\alpha \in \Gamma}$ satisfy 2.1 then $(\mathscr{R}(X), \rho)$, with * and ' properly defined, becomes the desired partial Baer $*$-semigroup of relations. Condition (AS) is used to prove the required associativity of $\mathscr{R}(X)$ and (UP) is needed to guarantee the uniqueness of the map '. It will be shown by examples that if either (AS) or (UP) are not satisfied then $\mathscr{R}((X)$ need not be a partial Baer $*$-semigroup. Also by the same examples we will see that conditions (AS) and (UP) are independent.

Although condition (UP) does guarantee the uniqueness of ', a comment concerning its explicit use is needed. It is used in the following manner. Suppose that $\left(M^{c}, T\right) \in \rho$ with $T \in \mathscr{R}_{1}(X)$ and $M^{c} \in \mathscr{R}_{2}(X)$. Since (UP) holds, $\left(M^{c}, T\right) \in \rho$ implies that there exists an $\alpha \in \Gamma$ such that $M,(\operatorname{dom} T) \subseteq X_{\alpha}$. Observe that if (UP) did not hold then we could only conclude that there exists $\beta \in \Gamma$ and an $N \subseteq X_{\beta}$ with $M^{c}=N^{c}$ such that $N,(\operatorname{dom} T) \subseteq X_{\beta}$. Siurilar deductions are made by using (UP) when either $\left(T, M^{c}\right)$ or $\left(M^{c}, N^{c}\right)$ belong to $\rho$ with $T \in \mathscr{R}_{1}(X)$ and $M^{c}, N^{c} \in \mathscr{R}_{2}(X)$. In the sequel no further mention of this use of (UP) will be made.

Lemma 2.2. Let $\left\{X_{\alpha}\right\}_{\alpha \in \Gamma}$ be as given in 2.1. Then $\left(\mathscr{R}(X), \rho,{ }^{*}\right)$ is a partial involution semigroup with the empty set $\varnothing$ as the zero.

Proof. Observe that if $(S, T) \in \rho$ then $S T \in \mathscr{R}(X)$. In fact $S T \in \mathscr{R}_{1}(X)$ unless $S, T \in \mathscr{R}_{2}(X)$ in which case $S T \in \mathscr{R}_{2}(X)$. Since it is immediate that $*$ is an involution and that $\varnothing$ is the zero, we need only show that $(\mathscr{R}(X), \rho)$ is a partial semigroup. To do this first let $(S, T),(T, U),(S T, U) \in \rho$. We show that $(S, T U) \in \rho$ by considering five separate cases.

Case, 1. Let $S, U \in \mathscr{R}(X)$ and $T \in \mathscr{R}_{1}(X)$. Since $(T, U) \in \rho, T U$ is defined and the definition of product gives $(\operatorname{dom} T U) \subseteq(\operatorname{dom} T)$. But $(S, T) \in \rho$ implies that there exists an $\alpha \in \Gamma$ such that $(\operatorname{ran} S),(\operatorname{dom} T) \subseteq X_{\alpha}$ when $S \in \mathscr{R}_{1}(X)$ and such that $Q,(\operatorname{dom} T) \subseteq X_{\alpha}$ for $S=Q^{c} \in \mathscr{R}_{2}(X)$. Hence either $(\operatorname{ran} S),(\operatorname{dom} T U)$ or $Q,(\operatorname{dom} T U)$ is contained in $X_{\alpha}$ so that $(S, T U) \in \rho$.

Case 2. Let $S, U \in \mathscr{R}_{1}(X)$ and $T=M^{c} \in \mathscr{R}_{2}(X)$. Since $(S, T),(T, U) \in \rho$ there exists $\alpha, \beta \in \Gamma$ such that $(\operatorname{ran} S), M \subseteq X_{\alpha}$ and $(\operatorname{dom} U), M \subseteq X_{\beta}$. From the definition of $M^{c}$, the fact that $M,(\operatorname{ran} S) \subseteq X_{\alpha}$, and the definition of the product $S T$, we have

$$
(\operatorname{ran} S T)=(\operatorname{ran} S) \cap\left(X_{\alpha} \backslash M\right)=(\operatorname{ran} S) \backslash M .
$$

But $(S T, U) \in \rho$ implies that there exists a $\gamma \in \Gamma$ such that $(\operatorname{ran} S T),(\operatorname{dom} U) \subseteq X_{\gamma}$. Therefore $(\operatorname{ran} S) \backslash M$, (dom $U) \subseteq X_{\gamma}$ so that by condition (AS) on $\left\{X_{\mu}\right\}_{\mu \in \Gamma}$ there 
exists a $\delta \in \Gamma$ such that $(\operatorname{ran} S),(\operatorname{dom} U) \backslash M \subseteq X_{\delta}$. But $M,(\operatorname{dom} U) \subseteq X_{\beta}$ so that

$$
(\operatorname{dom} T U)=(\operatorname{dom} U) \cap\left(X_{\beta} \backslash M\right)=(\operatorname{dom} U) \backslash M .
$$

Hence $(\operatorname{dom} T U),(\operatorname{ran} S) \subseteq X_{\delta}$ from which $(S, T U) \in \rho$.

Case 3. Let $S=Q^{c}, T=M^{c} \in \mathscr{R}_{2}(X)$ and $U \in \mathscr{R}_{1}(X)$. Since $\left(Q^{c}, M^{c}\right) \in \rho$ either $Q \subseteq M$ or $M \subseteq Q$. First assume $Q \subseteq M$. Since $\left(M^{c}, U\right) \in \rho$, there exists an $\alpha \in \Gamma$ such that $M,(\operatorname{dom} U) \subseteq X_{\alpha}$. Also $\left(\operatorname{dom} M^{c} U\right) \subseteq(\operatorname{dom} U)$. Therefore $\left(Q^{c}, M^{c} U\right) \in \rho$. Next assume $M \subseteq Q$. Then $Q^{c} M^{c}=Q^{c}$. Now $\left(Q^{c} M^{c}, U\right)$ $=\left(Q^{c}, U\right) \in \rho$ so that there exists a $\gamma \in \Gamma$ such that $Q,(\operatorname{dom} U) \subseteq X_{\gamma}$. But $\left(\operatorname{dom} M^{c} U\right) \subseteq(\operatorname{dom} U)$ from which $Q,\left(\operatorname{dom} M^{c} U\right) \subseteq X_{\gamma}$. Hence $\left(Q^{c}, M^{c} U\right) \in \rho$.

Case 4. Let $S \in \mathscr{R}_{1}(X)$ and $T=M^{c}, U=N^{c} \in \mathscr{R}_{2}(X)$. Since $\left(M^{c}, N^{c}\right) \in \rho$ either $M \subseteq N$ or $N \subseteq M$. If $N \subseteq M$ then $M^{c} N^{c}=M^{c}$ and hence $\left(S, M^{c} N^{c}\right)$ $=\left(S, M^{c}\right) \in \rho$ by hypothesis. Therefore we may assume $M \subseteq N$. Now $\left(S, M^{c}\right) \in \rho$ implies that there exists an $\alpha \in \Gamma$ such that $(\operatorname{ran} S), M \subseteq X_{\alpha}$ from which $\left(\operatorname{ran} S M^{c}\right)$ $=(\operatorname{ran} S) \backslash M$. Also $\left(M^{c}, N^{c}\right) \in \rho$ with $M \subseteq N$ implies $M^{c} N^{c}=N^{c}$ while $\left(S M^{c}, N^{c}\right) \in \rho$ implies the existence of $\beta \in \Gamma$ such that $\left(\operatorname{ran} S M^{c}\right) \subseteq X_{\beta}$ and $M \subseteq N \subseteq X_{\beta}$. Therefore

$$
(\operatorname{ran} S) \subseteq((\operatorname{ran} S) \backslash M) \cup M=\left(\operatorname{ran} S M^{c}\right) \cup M \subseteq X_{\beta}
$$

so that (ran $S), N \subseteq X_{\beta}$ and thus $\left(S, N^{c}\right)=\left(S, M^{c} N^{c}\right) \in \rho$.

Case 5. Let: $S=Q^{c}, \quad T=M^{c}, \quad U=N^{c} \in \mathscr{R}_{2}(X) . \quad\left(Q^{c}, M^{c}\right),\left(M^{c}, N^{c}\right) \in \rho$ imply $[Q \subseteq M$ or $M \subseteq Q]$ and $[M \subseteq N$ or $N \subseteq M]$. If $N \subseteq M$ then $M^{c} N^{c}=M^{c}$ so that $\left(Q^{c}, M^{c} N^{c}\right)=\left(Q^{c}, M^{c}\right) \in \rho$ by hypothesis. Therefore assume $M \subseteq N$. If $Q \subseteq M \subseteq N$, then $M^{c} N^{c}=N^{c}$ and $Q \subseteq M$ so $\left(Q^{c}, N^{c}\right)=\left(Q^{c}, M^{c} N^{c}\right) \in \rho$. If $M \subseteq Q$ and $M \subseteq N$ then since $\left(Q^{c} M^{c}, N^{c}\right)=\left(Q^{c}, N^{c}\right) \in \rho$ and $M^{c} N^{c}=N^{c}$ we have $\left(Q^{c}, M^{c} N^{c}\right) \in \rho$.

To complete the proof that $(\mathscr{R}(X), \rho)$ is a partial semigroup we next let $(S, T),(T, U),(S, T U) \in \rho$. Since $*$ is an involution on $\mathscr{R}(X)$ it follows that $\left(T^{*}, S^{*}\right),\left(U^{*}, T^{*}\right),\left(U^{*} T^{*}, S^{*}\right) \in \rho$. By the part just proved $\left(U^{*}, T^{*} S^{*}\right) \in \rho$ so that applying * we obtain $(S T, U) \in \rho$ as desired. Finally since our definition of partial product is composition of relations and composition of relations is associative, we have that $(S, T),(T, U),(S, T U),(S T, U) \in \rho$ implies $(S T) U=S(T U)$ and the proof is complete.

LeMmA 2.3. Let $\left\{X_{\alpha}\right\}_{\alpha \in \Gamma}$ be as given in 2.1. For $S \in \mathscr{R}(X)$ there exists a projection $S^{\prime} \in \mathscr{R}(X)$ such that when $T \in \mathscr{R}(X)$ we have $(S, T) \in \rho$ and $S T=\varnothing$ if and only if $\left(S^{\prime}, T\right) \in \rho$ and $T=S^{\prime} T$.

Proof. We prove the lemma by considering two cases.

Case 1. Let $S \in \mathscr{R}_{1}(X)$. We show that for this case $S^{\prime}=(\operatorname{ran} S)^{c}$. Notice that since $S \in \mathscr{R}_{1}(X)$ there exists an $\alpha \in \Gamma$ such that $(\operatorname{ran} S) \subseteq X_{\alpha}$ so that $(\operatorname{ran} S)^{c}$ makes sense. Also from its definition $(\operatorname{ran} S)^{c}$ is a projection. 
Let $(S, T) \in \rho$ and $S T=\varnothing$. First assume that $T \in \mathscr{R}_{1}(X)$. Now $T \in \mathscr{R}_{1}(X)$ and $S T=\varnothing$ implies that there exists an $\alpha \in \Gamma$ such that $(\operatorname{ran} S),(\operatorname{dom} T) \subseteq X_{\alpha}$ with $(\operatorname{ran} S) \cap(\operatorname{dom} T)=\varnothing$. Hence from the definition of $K(\operatorname{ran} S)$ we have that $(\operatorname{dom} T) \subseteq K(\operatorname{ran} S)$. If $(\operatorname{ran} S)^{c} \in \mathscr{R}_{1}(X)$ then there exists a $\beta \in \Gamma$ such that $(\operatorname{dom} T) \subseteq K(\operatorname{ran} S) \subseteq X_{\beta}$ from which $\left((\operatorname{ran} S)^{c}, T\right) \in \rho$ with $T=(\operatorname{ran} S)^{c} T$, while if $(\operatorname{ran} S)^{c} \in \mathscr{R}_{2}(X)$ then since $(\operatorname{ran} S),(\operatorname{dom} T) \subseteq X_{\alpha}$ we again have $\left((\operatorname{ran} S)^{c}, T\right) \in \rho$ with $T=(\operatorname{ran} S)^{c} T$. Next assume $T=M^{c} \in \mathscr{R}_{2}(X)$. Then since $(S, T) \in \rho$ we have that there exists an $\alpha \in \Gamma$ such that $M,(\operatorname{ran} S) \subseteq X_{\alpha}$. Also $S M^{c}=\varnothing$ implies from the definition of $M^{c}$ that $(\operatorname{ran} S) \cap\left(X_{\alpha} \backslash M\right)=\varnothing$ and hence, since $(\operatorname{ran} S) \subseteq X_{\alpha},(\operatorname{ran} S) \subseteq M \subseteq X_{\alpha}$. Therefore $K(M) \subseteq K(\operatorname{ran} S)$ so that $M^{c} \subseteq(\operatorname{ran} S)^{c}$. Now $(\operatorname{ran} S)^{c} \in \mathscr{R}_{2}(X)$ for otherwise $M^{c} \subseteq(\operatorname{ran} S)^{c}$ would imply that $M^{c} \in \mathscr{R}_{1}(X)$, a contradiction to our assumption. Hence $(\operatorname{ran} S) \subseteq M \subseteq X_{\alpha}$ and $\left((\operatorname{ran} S)^{c}, M^{c}\right) \in \rho$ with $M^{c}=(\operatorname{ran} S)^{c} M^{c}$.

Conversely, let $\left((\operatorname{ran} S)^{c}, T\right) \in \rho$ with $T=(\operatorname{ran} S)^{c} T$. We first claim that $\left(\mathrm{S},(\operatorname{ran} S)^{c}\right) \in \rho$. To see this suppose the claim does not hold. Then from the definition of $\rho$ there exists a $\gamma \in \Gamma$ such that $K(\operatorname{ran} S) \subseteq X_{\gamma}$ but either $(\operatorname{ran} S) \notin X_{\alpha}$ or $K(\operatorname{ran} S) \nsubseteq X_{\alpha}$ for all $\alpha \in \Gamma$. Hence there must exist $\beta, \delta \in \Gamma, \beta \neq \delta$, such that $(\operatorname{ran} S) \subseteq X_{\beta} \cap X_{\delta}$. Thus since $K(\operatorname{ran} S) \subseteq X_{\gamma}$, we have $\left.X_{\beta}\right)(\operatorname{ran} S), X_{\delta} \mid(\operatorname{ran} S) \subseteq X_{\gamma}$ which implies by condition (AS) that there exists a $\mu \in \Gamma$ such that $X_{\beta}, X_{\delta} \mid(\operatorname{ran} S)$ $\subseteq X_{\mu}$. But $X_{\beta} \subseteq X_{\mu}$ implies $\beta=\mu$ so that $X_{\delta} \backslash(\operatorname{ran} S) \subseteq X_{\beta}$. Therefore since $(\operatorname{ran} S) \subseteq X_{\beta}$,

$$
X_{\delta}=\left(X_{\delta} \backslash \operatorname{ran} S\right) \cup(\operatorname{ran} S) \subseteq X_{\hat{p}}
$$

which implies that $\beta=\delta$, a contradiction. Hence the supposition is false and $\left(S,(\operatorname{ran} S)^{c}\right) \in \rho$. Since $(\operatorname{ran} S) \cap K(\operatorname{ran} S)=\varnothing$ it is clear that $S(\operatorname{ran} S)^{c}=\varnothing$. Therefore since $(\mathscr{R}(X), \rho)$ is a partial semigroup and $\left((\operatorname{ran} S)^{c}, T\right),\left(S,(\operatorname{ran} S)^{c}\right) \in \rho$ with

$$
T=(\operatorname{ran} S)^{c} T \text { and } S(\operatorname{ran} S)^{c}=\varnothing,
$$

we have $(\varnothing, T)=\left(S(\operatorname{ran} S)^{c}, T\right) \in \rho$ and thus $\left(S,(\operatorname{ran} S)^{c} T\right)=(S, T) \in \rho$ with $S T=\varnothing$.

Case 2. Let $S=M^{c} \in \mathscr{R}_{2}(X)$ where $M \subseteq X_{\alpha}$ for some $\alpha \in \Gamma$. For: this case we show

$$
S^{\prime}=\Delta_{X} \cap(M \times M) .
$$

For convenience we denote $\Delta_{X} \cap(M \times M)$ by $W$. It is clear that $W$ is a projection.

Let $\left(M^{c}, T\right) \in \rho$ with $M^{c} T=\varnothing$. If $T \in \mathscr{R}_{1}(X)$ there exists an $\alpha \in \Gamma$ such that $(\operatorname{dom} T), M \subseteq X_{\alpha}$ and $K(M) \cap(\operatorname{dom} T)=\varnothing$, that is, $(\operatorname{dom} T) \subseteq M \subseteq X_{\alpha}$. But $(\operatorname{dom} T) \subseteq M=(\operatorname{ran} W)$ so that $(W, T) \in \rho$ and $T=W T$. If $T=N^{c} \in \mathscr{R}_{2}(X)$ then $\left(M^{c}, N^{c}\right) \in \rho$ with $M^{c} N^{c}=\varnothing$ so that either $M^{c}=\varnothing$ or $N^{c}=\varnothing$, a contradiction to $M^{c}, N^{c} \in \mathscr{R}_{2}(X)$. Hence $\left(M^{c}, T\right) \in \rho$ with $M^{c} T=\varnothing$ implies $T \in \mathscr{R}_{1}(X)$ and $(W, T) \in \rho$ with $T=W T$. 
Conversely if $(W, T) \in \rho$ and $T=W T$ then since $\left(M^{c}, W\right),(W, T),\left(M^{c} W, T\right)$ $=(\varnothing, T) \in \rho$, we have $\left(M^{c}, W T\right)=\left(M^{c}, T\right) \in \rho$ with $M^{c} T=\varnothing$.

We summarize Lemmas 2.2 and 2.3 in the following theorem.

THEOREM 2.4. $\left(\mathscr{R}(X), \rho, *,{ }^{\prime}\right)$ is a partial Baer *-semigroup whenever the family $\left\{X_{\alpha}\right\}_{\alpha \in \Gamma}$ satisfies 2.1 and 'is as defined in 2.3.

Except for the fact that $P^{\prime}(S)$ will still denote the closed projections of a partial Baer *-semigroup $S$, in the sequel the symbol'will be restricted to the mapping given in 2.3.

Observe that when $\Gamma=\{\alpha\}$ and $X=X_{\alpha}$, then the partial Baer *-semigroup $\mathscr{R}(X)$ of 2.4 is simply the Baer ${ }^{*}$-semigroup of relations $\mathscr{P}(X \times X)$ of Foulis (unpublished). Also for any family $\left\{X_{\alpha}\right]_{\alpha \in \Gamma}$ of 2.1 each Baer ${ }^{*}$-semigroup $\mathscr{R}\left(X_{\alpha}\right)=$ $\mathscr{P}\left(X_{\alpha} \times X_{\alpha}\right), \alpha \in \Gamma$, is contained in $\mathscr{R}(X)$ with $\mathscr{R}\left(X_{\alpha}\right) \times \mathscr{R}\left(X_{\alpha}\right) \leqq \rho$.

We close this section by considering two examples which indicate why conditions (AS) and (UP) are used in our construction of $\mathscr{R}(X)$. These examples also show the independence of (AS) and (UP).

EXAMPLE 2.5. Let $X_{1}=\{a, b, d\}, X_{2}=\{a, e\}, X_{3}=\{b, e\}$, with $\Gamma=\{1,2,3\}$.

EXAMPLE 2.6. Let $X_{1}=\{a, b, d, e\}, \quad X_{2}=\{d, e, f, g\}, \quad X_{3}=\{f, g, h, j\}$, $X_{4}=\{h, j, a, b\}$ with $\Gamma=\{1,2,3,4\}$.

We first claim that the sets $\left\{X_{i}\right\}_{i=1,2,3}$ of 2.5 do not satisfy (AS) and in addition that $\mathscr{R}(X)$, for this example, is not even a partial semigroup. To show this choose $M=\{a\}, N=\{a, b\}$, and $Q=\{e\}$. It is apparent that $M \subseteq X_{1} \cap X_{2}$, $N \subseteq X_{1}, Q \subseteq X_{2}$, and $N \backslash M, Q \subseteq X_{3}$, but there does not exist a $\delta \in \Gamma$ such that $N, Q \backslash M \subseteq X_{\delta}$. Therefore (AS) is not satisfied. To see that $(\mathscr{R}(X), \rho)$ is not a partial semigroup first set $S=N \times N, U=Q \times X_{3}$. By definition

$$
M^{c}=\Delta_{X} \cap(\{b, d, e\} \times\{b, d, e\})
$$

and we have $\left(S, M^{c}\right),\left(M^{c}, U\right),\left(S M^{c}, U\right) \in \rho$ with $S M^{c}=N \times\{b\}$. But $U=M^{c} U$ and there does not exist a $\delta \in \Gamma$ such that $N, Q \subseteq X_{\delta}$. Hence $\left(S, M^{c} U\right) \notin \rho$ and $(\mathscr{R}(X), \rho)$ is not a partial semigroup. It is immediate that these sets do satisfy (UP).

Next we examine 2.6. It is straightforward to check that the sets of this example do satisfy (AS) while $\{d, e\}^{c}=\{h, j\}^{c}$ implies that (UP) fails to hold. Also by inspection there is no projection $W \in \mathscr{R}(X)$ such that $\left(\{d, e\}^{c}, T\right) \in \rho$ with $\{d, e\}^{c} T$ $=\varnothing$ if and only if $(W, T) \in \rho$ with $T=W T$. Hence $\mathscr{R}(X)$, for this example, also fails to be a partial Baer *-semigroup.

\section{A class of orthomodular posets coordinatized by $\mathscr{R}(X)$}

In the previous section we have seen that $\mathscr{R}(X)$ is, for $\left\{X_{\alpha}\right\}_{\alpha \in \Gamma}$ properly chosen, a partial Baer *-semigroup generalizing Foulis' Baer *-semigroup of 
relations. We now wish to show further that $\mathscr{R}(X)$ coordinatizes certain orthomodular posets which are "Greechie pastings" of the complete atomic Boolean

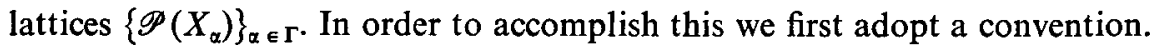

Convention 3.1. Let $(P, \leqq p, \#)$ be an orthocomplemented poset such that

(1) $P=\bigcup_{\alpha \in \Gamma} B_{\alpha}$ where $\left(B_{\alpha}, \leqq_{\alpha} \#_{\alpha}\right)$ is a complete atomic [Boolean lattice for all $\alpha \in \Gamma$,

(2) if $\alpha \neq \beta(\alpha, \beta \in \Gamma)$ then $B_{\alpha} \cap B_{\beta}=\{0,1\}$ or $\left\{0,1, a, a^{\#}\right\}$ where $a$ is an atom of both $B_{\alpha}$ and $B_{\beta}, a^{\#}=a^{\# \alpha}=a^{\# \beta}$,

(3) $B_{\alpha} \nsucceq 2^{1}, B_{\alpha} \nsucceq 2^{2}$ for all $\alpha \in \Gamma$, where $2^{n}$ denotes the Boolean lattice of all subsets of an $n$ element set,

(4) for $x, y \in P, x \leqq_{P} y$ if and only if there exists an $\alpha \in \Gamma$ such that $\{x, y\} \subseteq B_{a}$ and $x \leqq_{B_{\alpha}} y$, and

(5) for $x \in P$ there exists an $\alpha \in \Gamma$ such that $x \in B_{\alpha}$ and $x^{\#}=x^{\# \alpha}$.

Greechie (1971) calls a finite set $\left\{B_{i}\right\}_{i=1,2 \ldots, n}$, each $B_{i} \in\left\{B_{\alpha}\right\}_{\alpha \in \Gamma}$ of 3.1 , an atomistic loop of order $n$ whenever for $1 \leqq j<i \leqq n$

$$
B_{i} \cap B_{j}= \begin{cases}\left\{0,1, a, a^{*}\right\} & \text { if } i-j \in\{1, n-1\} \\ \{0,1\} & \text { otherwise, }\end{cases}
$$

and for $1 \leqq k<j<i \leqq n$ we have $B_{i} \cap B_{j} \cap B_{k}=\{0,1\}$. Furthermore he shows that the orthocomplemented poset $\left(P, \leqq_{P}, \#\right)$ of 3.1 is orthomodular if and only if all atomistic loops of $P$ are at least of order four. Thus we put additional stipulations on 3.1 .

Convention 3.2. Let $\left(P, \leqq_{P}, \#\right)$ and $\left\{B_{\alpha}\right\}_{\alpha \in \Gamma}$ be as in 3.1 such that

(1) all atomistic loops of $P$ are at least of order four, and

(2) $X_{\alpha}$ is defined as the set of atoms of $B_{\alpha}, \alpha \in \Gamma$, with $X=\bigcup_{\alpha \in \Gamma} X_{\alpha}$.

Observe that $X=$ atoms of $P$ and that $X_{\alpha} \ddagger X_{\beta}$ for $\alpha \neq \beta$ in $\Gamma$ (see (2) and (3) of 3.1).

LEMma 3.3. The sets $\left\{X_{\alpha}\right\}_{\alpha \in \Gamma}$ of 3.2 satisfy condition (AS) of 2.1.

Proof. The proof is by contradiction. Suppose there exist $\alpha, \beta, \gamma \in \Gamma$ and sets $M, N, Q$ such that $M \subseteq X_{\alpha} \cap X_{\beta}, N \subseteq X_{\alpha}, Q \subseteq X_{\beta}$, and $N \backslash M, Q \subseteq X_{\gamma}$ but $N \nsubseteq X_{\delta}$ or $Q \backslash M \nsubseteq X_{\delta}$ for all $\delta \in \Gamma$. Notice that this supposition implies that $\alpha, \beta, \gamma$ are distinct indices. Now $N \backslash M \subseteq X_{\gamma}$ and $N \nsubseteq X_{\gamma}$ imply that there exists an $m_{1} \in M$ with $m_{1} \notin X_{\gamma}$. Also $M \subseteq X_{\beta}, N \nsubseteq X_{\beta}$ imply the existence of an $m_{2} \in N \backslash M$ with $m_{2} \notin X_{\beta}$ while $Q \backslash M \nsubseteq X_{\alpha}$ implies there exists an $m_{3} \in Q \backslash M$ with $m_{3} \notin X_{\alpha}$. Observe that $m_{1} \in X_{\alpha} \cap X_{\beta}, m_{2} \in X_{\alpha} \cap X_{\gamma}$, and $m_{3} \in X_{\beta} \cap X_{\gamma}$. Therefore $\left\{m_{1}, m_{2}, m_{3}\right\}$ are distinct atoms and $\left\{B_{\alpha}, B_{\beta}, B_{\gamma}\right\}$ form an atomistic loop of order three, a contradiction to (1) of 3.2. Hence the supposition is false and (AS) holds. 
LEMMA 3.4. The sets $\left\{X_{\alpha}\right\}_{\alpha \in \Gamma}$ of 3.2 satisfy condition (UP) of 2.1 .

Proof. Suppose here exist $\alpha, \beta \in \Gamma$ and sets $M \subseteq X_{\alpha}, N \subseteq X_{\beta}$ such that $K(N)=K(M)$ with $K(M) \ddagger X_{\gamma}, \gamma \in \Gamma$. Since $K(M) \ddagger X_{\gamma}, \gamma \in \Gamma$, we have $|\Gamma(M)|>1$. By (2) of $3.1 M=\varnothing$ or $\{a\}$ where $a \in X$. Clearly $K(M)=K(N)=X$ if and only if $M=N=\varnothing$ so that we may assume $M=\{a\}$ and $N=\{b\}$ with $a, b \in X$. (UP) will be established by showing $a=b$. Suppose $a \neq b$ and let $\alpha \in \Gamma$ such that $a \in X_{\alpha}$. Since $B_{\alpha} \not 2^{1}$ there exists

$$
d \in X_{a} \backslash\{a\} \subseteq K(M)=K(N) .
$$

Thus there exists a $\beta \in \Gamma$ such that $b, d \in X_{\beta}$. Surely $\beta \neq \alpha$, since $\beta=\alpha$ would imply

$$
b \in X_{\beta} \backslash\{a\} \subseteq K(M)=K(N),
$$

a contradiction to the definition of $K(N)$. Since $B_{\beta} \not 2^{2}$ there exists an $e \in X_{\beta} \backslash\{b, d\}$. Now $e \notin X_{\alpha}$ for otherwise $\{d, e\} \subseteq X_{\alpha} \cap X_{\beta}$ which violates (2) of 3.1. But

$$
e \in X_{\beta} \backslash\{b\} \subseteq K(N)=K(M)
$$

implies the existence of a $\gamma \in \Gamma$ such that $e, a \in X_{\gamma}$. Clearly $\gamma \neq \alpha$ for $\gamma=\alpha$ would again imply $\{d, e\} \subseteq X_{\alpha} \cap X_{\beta}$. Also $\gamma \neq \beta$ since $\gamma=\beta$ would imply $a, b \in X_{\beta}$ violating $K(M)=K(N)$. Hence $\alpha, \beta, \gamma$ are all distinct with $d \in X_{\alpha} \cap X_{\beta}, e \in X_{\beta} \cap X_{\gamma}$, and $a \in X_{\alpha} \cap X_{\gamma}$ so that $\left\{B_{\alpha}, B_{\beta}, B_{\gamma}\right\}$ is an atomistic loop, a contradiction to (1) of 3.2. Thus $a=b$ and (UP) holds.

From 2.4, 3.3, and 3.4 we have the following theorem.

THeOREM 3.5. If $P,\left\{X_{\alpha}\right\}_{\alpha \in \Gamma}$, and $X$ are as in 3.2 , then $\left(\mathscr{R}(X), \rho,{ }^{*},{ }^{\prime}\right)$ is a partial Baer *-semigroup.

The fact that $\mathscr{R}(X)$ of Theorem 3.5 is just a partial Baer *-semigroup is not satisfactory. We wish in addition to show that it coordinatizes $P$. This is done by proving a sequence of lemmas. In the se lemmas and their proofs we retain the notation of 3.1 and 3.2 and in addition for $x \in P$ let $A_{x}=\left\{a \mid a \leqq{ }_{P} x\right.$ and $\left.a \in X\right\}$, that is, $A_{x}$ denotes all the atoms in $P$ under $x$.

We first summarize some needed facts.

LEMMA 3.6.

(i) Let $x \in P$ with $\Gamma\left(A_{x}\right)=\{\alpha\}, \alpha \in \Gamma$. Then

(a) $\Gamma\left(A_{x} \#\right)=\{\alpha\}$,

(b) $A_{x} \#=X_{\alpha} \backslash A_{x}$

(c) $\left(A_{x}\right)^{c}=\Delta_{X} \cap\left(A_{x} \# \times A_{x} \#\right)=\left[\left(A_{x} \#\right)^{c}\right]^{\prime}$, and

(d) $\left(A_{x} \#\right)^{c}=\Delta_{X} \cap\left(A_{x} \times A_{x}\right)=\left[\left(A_{x}\right)^{c}\right]^{\prime}$.

(ii) (a) $A_{1}=\bigcup_{\alpha \in \Gamma} X_{\alpha}$,

(b) $A_{0}=\varnothing$, 
(c) $\varnothing^{c}=\left[\Delta_{X} \cap(\varnothing \times \varnothing)\right]^{\prime}=\Delta_{X} \cap\left(A_{1} \times A_{1}\right)$, and

(d) $\left[\varnothing^{c}\right]^{\prime}=\Delta_{X} \cap(\varnothing \times \varnothing)=\Delta_{X} \cap\left(A_{0} \times A_{0}\right)$.

(iii) Let $y$ be an atom of $P$. Then

(a) $A_{y}=\{y\}$,

(b) $A_{y} \#=\bigcup_{\alpha \in \mathrm{r}([y])}\left(X_{\alpha} \backslash\{y\}\right)$,

(c) $A_{y}{ }^{c}=\Delta_{X} \cap\left(A_{y} \# \times A_{y} \#\right)=\left[\Delta_{x} \cap\left(A_{y} \times A_{y}\right)\right]^{\prime}$, and

(d) $\left[A_{y}{ }^{c}\right]^{\prime}=\Delta_{X} \cap\left(A_{y} \times A_{y}\right)$.

Proof. All parts of lemma are immediate upon recollection

$$
\text { that } S^{\prime}=\left\{\begin{array}{l}
(\operatorname{ran} S)^{c} \text { for } S \in \mathscr{R}_{1}(X) \\
\Delta_{X} \cap(M \times M) \text { for } S=M^{c} \in \mathscr{R}_{2}(X)
\end{array}\right. \text { and }
$$

restrictions imposed on $\left\{X_{\alpha}\right\}_{\alpha \in \Gamma}$ in 3.1 and 3.2.

Lemma 3.7. Let $x \in P$. Then $\Delta_{X} \cap\left(A_{x} \times A_{x}\right) \in P^{\prime}(\mathscr{R}(X))$, that is, is a closed projection of $\mathscr{R}(X)$.

Proof. Recall that $S \in P^{\prime}(\mathscr{R}(X))$ if and only if $S=T^{\prime}$ for some $T \in \mathscr{R}(X)$. First the result is immediate by 3.6 (i) and (ii) for $x=0,1$ or when $\left|\Gamma\left(A_{x}\right)\right|=1$. Therefore we assume that $x \neq 0,1$ and that either $A_{x} \notin X_{\alpha}$ for all $\alpha \in \Gamma$ or $\left|\Gamma\left(A_{x}\right)\right|>1$. But by (2) of 3.1 this implies that $x$ is either an atom or coatom of $P$. By 3.6 (iii) we again obtain

$$
\Delta_{X} \cap\left(A_{x} \times A_{x}\right) \in P^{\prime}(\mathscr{R}(X))
$$

and the proof is complete.

Having established 3.7 we now define a map $f: P \rightarrow P^{\prime}(\mathscr{R}(X))$ by the prescription

$$
x f=\Delta_{X} \cap\left(A_{x} \times A_{x}\right), \quad x \in P .
$$

We will in fact show that $f$ is an order isomorphism onto $\left(P^{\prime}(\mathscr{R}(X))\right.$, $\left.\leqq,{ }^{\prime}\right)$ preserving orthocomplementation, i.e., that $\mathscr{R}(X)$ coordinatizes $P$.

LEMMA 3.8. $f$ maps onto $P^{\prime}(\mathscr{R}(X))$.

Proof. By the proof of $2.3 P^{\prime}(\mathscr{R}(X))=\mathscr{A} \cup \mathscr{C}$ where

$$
\mathscr{A}=\left\{(\operatorname{ran} T)^{c} \mid T \in \mathscr{R}_{1}(X)\right\} \text { and } \mathscr{C}=\left\{\Delta_{X} \cap(M \times M) \mid M^{c} \in \mathscr{R}_{2}(X)\right\} .
$$

Let $S \in P^{\prime}(\mathscr{R}(X))$. If $S \in \mathscr{C}$, then there exists an $\alpha \in \Gamma$ and an $M \subseteq X_{\alpha}$ such that $S=\Delta_{X} \cap(M \times M)$ with $|\Gamma(M)|>1$. Thus by (2) of $3.1 M=\varnothing$ or $M=\{a\}$, $a \in X$. But $0 f=\Delta_{X} \cap(\varnothing \times \varnothing)$ and $a f=\Delta_{X} \cap(\{a\} \times\{a\}), a \in X$, so that $\mathscr{C}$ is contained in the range of $f$. We thus assume that there exists a $T \in \mathscr{R}_{1}(X)$ such that $S=(\operatorname{ran} T)^{c}$, i.e., that $S \in \mathscr{A} . T \in \mathscr{R}_{1}(X)$ implies that there exists 
$\gamma \in \Gamma$ such that $(\operatorname{ran} T) \subseteq X_{\gamma}$. Hence, since by $3.1(1) B_{\gamma}$ is complete, $V_{B_{\gamma}}(\operatorname{ran} T)$ exists. We claim that $\left(x^{\#}\right) f=(\operatorname{ran} T)^{c}=S$ where $x=\vee_{B_{\gamma}}(\operatorname{ran} T)$. Now this claim is immediate if $(\operatorname{ran} T)=\varnothing, X_{\gamma}$, or $X_{\gamma} \backslash\{a\}, a \in X_{\gamma}$. Also if $(\operatorname{ran} T)=\{a\}$, $a \in X_{\gamma}$, then $x=a$ and by 3.6 (iii)

$$
\left(x^{\#}\right) f=\Delta_{X} \cap\left(A_{x^{*}} \times A_{x^{*}}\right)=A_{x}^{c}=(\operatorname{ran} T)^{c} .
$$

But if (ran $T) \neq \varnothing, X_{\gamma}, X_{\gamma} \backslash\{a\},\{a\}$ for $a \in X_{\gamma}$ it follows from (2) of 3.1 that $\operatorname{ran} T=A_{x}$ and $\Gamma(\operatorname{ran} T)=\{\gamma\}$.Under these conditions $3.6(\mathrm{i})$ implies

$$
\left(x^{\#}\right) f=\Delta_{\mathrm{X}} \cap\left(A_{x^{*}} \times A_{x^{*}}\right)=A_{x}^{c}=(\operatorname{ran} T)^{c}
$$

and the claim holds.

LEMMA 3.9. $f$ is a one-to-one map with $f$ and $f^{-1}$ preserving order on $P$ and $P^{\prime}(\mathscr{R}(X))$ respectively.

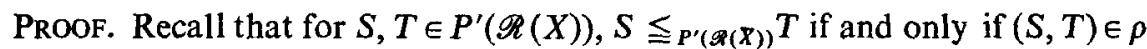
and $S=S T$. Let $x \leqq_{P} y, x, y \in P$. Since

$$
x f=\Delta_{X} \cap\left(A_{x} \times A_{x}\right) \text { and } y f=\Delta_{X} \cap\left(A_{y} \times A_{y}\right),
$$

it is straightforward to check that $(x f, y f) \in \rho$. Thus since $A_{x} \subseteq A_{y}$ we have tha $x f=(x f)(y f$ and $f$ preserves order.

Next let $S=\Delta_{X} \cap(M \times M), \quad T=\Delta_{X} \cap(Q \times Q) \in P^{\prime}(\mathscr{R}(X))$ with $(\mathrm{S}, T) \in \rho$ and $S=S T$. Thus $M \subseteq Q$ and by 3.8 both $S f^{-1}$ and $T f^{-1}$ are nonempty. Let $x \in S f^{-1}$ and $y \in T f^{-1}$ and observe that

$$
M=\left\{a \mid a \leqq_{P} x, a \in X\right\} \text { and } Q=\left\{a \mid a \leqq_{P} y, a \in X\right\} .
$$

If $x \in M \subseteq Q$ then clearly $x \leqq_{P} y$ so that we may assume $x \notin M$. Letting $\beta \in \Gamma$ with $x \in B_{\beta}$ and $D=\left\{a \mid a \in M \cap B_{\beta}\right\}$ we obtain from the completeness of $B_{\beta}$ that $x=\vee_{B_{\beta}} D$. Since $x \notin M$, there exists and $a_{1} \neq a_{2}$ in $D$. We claim that this implies that $y \in B_{\beta}$ from which

$$
x=\vee_{B_{\beta}} D \leqq_{P} \vee_{B_{\beta}}\left(Q \cap X_{\beta}\right)=y .
$$

To see this claim first observe, since $a_{1}, a_{2} \in D \subseteq M \subseteq Q$, that (4) of 3.1 implies the existence of $\gamma, \delta \in \Gamma$ such that $a_{1} \leqq_{B_{\gamma}} y$ and $a_{2} \leqq_{B_{\delta}} y$. But if $y \in B_{\beta}$ then $\beta, \gamma, \delta$ are distinct with $y$ a coatom of both $B_{\gamma}$ and $B_{\delta}$ so that $\left\{B_{\beta}, B_{\gamma}, B_{\delta}\right\}$ forms an atomistic loop of order three, a contradiction. Hence $y \in B_{\beta}$ and we have $x \leqq_{P} y$.

Notice that the argument of the last paragraph gives in particular that $f$ is one-to-one. Thus since $f$ is onto, $f^{-1}$ exists on $P^{\prime}(\mathscr{R}(X))$. Hence both $f$ and $f^{-1}$ are one-to-one with $x \leqq_{p} y$ if and only if $x f \leqq_{p^{\prime}(X(X))} y f$.

LEMMA 3.10. $f$ preserves orthocomplementation.

Proof. Let $x \in P$. If $\left|\Gamma\left(A_{x}\right)\right|=1$ then by 3.6 (i) 


$$
\left(x^{\#}\right) f=\Delta_{X} \cap\left(A_{x^{*}} \times A_{x^{*}}\right)=\left[\left(A_{x^{*}}\right)^{c}\right]^{\prime}=\left[\Delta_{X} \cap\left(A_{x} \times A_{x}\right)\right]^{\prime}=[x f]^{\prime} .
$$

We may thus assume $\left|\Gamma\left(A_{x}\right)\right|>1$ or $A_{x} \notin X_{\alpha}$ for $\alpha \in \Gamma$, which says by (2) of 3.1 that $x \in\left\{0,1, a, a^{\#}\right\}$ where $a \in X$. But for $x \in\left\{0,1, a, a^{\#}\right\}, 3.6$ (ii) and (iii) give

$$
\left(x^{\#}\right) f=\Delta_{X} \cap\left(A_{x^{*}} \times A_{x^{*}}\right)=\left[\Delta_{\mathbf{X}} \cap\left(A_{x} \times A_{x}\right)\right]^{\prime}=(x f)^{\prime} .
$$

Hence in all cases $\left(x^{\#}\right) f=[x f]^{\prime}$.

We summarize the results of Lemmas 3.8 through 3.10 in the next theorem.

Theorem 3.11. Let $\left(P, \leqq_{P}, \#\right),\left\{X_{\alpha}\right\}_{\alpha \in \Gamma}$, and $X$ be as defined in Convention 3.2. Then the partial Baer *-semigroup $\left(\mathscr{R}(X), \rho, *,,^{\prime}\right)$ coordinatizes $\left(P, \leqq_{P}, \#\right)$.

\section{Examples}

The results of the previous section give a constructive method of obtaining non-trivial partial Baer *-semigroups which coordinatize many of the well known orthomodular posets. They are non-trivial in the sense that they are not the "minimal" ones discussed in Gudder and Schelp (1970). We look at several examples.

ExAmple 4.1. Let $X_{1}=\{a, b, c\}, X_{2}=\{c, d, e\}$, and $X_{3}=\{e, f, g\}$. For the collection $\left\{X_{i}\right\}_{i=1,2,3}, \mathscr{R}(X)$ coordinatizes $D_{16}$, the 16-element lattice of Dilworth (1940).

EXAMPLE 4.2. Let $X_{1}=(a, b, c\}, X_{2}=\{c, d, e\}, X_{3}=\{e, f, g\}$, and $X_{4}=\{g, h, a\}$. For this collection of sets $\mathscr{R}(X)$ coordinatizes $J_{18}$, the first known finite orthomodular poset which is not a lattice, see Janowitz (1963).

Example 4.3. Let $X_{1}=\{a, b, c\}, X_{2}=\{c, d, e\}, X_{3}=\{e, f, g\}$ $X_{4}=\{g, h, i\}, \quad X_{5}=\{i, j, k\}, \quad X_{6}=\{k, q, a\}, X_{7}=\{b, m, h\}, X_{8}=\{d, n, j\}$, $X_{9}=\{f, p, q\}, X_{10}=\{m, n, p\}$. For this collection of sets $\mathscr{R}(X)$ coordinatizes $G_{32}$, see [5], Greechie's 32-element orthomodular lattice. Greechie (1971).

EXAMPLE 4.4. Let $\left\{X_{\alpha}\right\}_{\alpha \in \Gamma}$ be a disjoint collection of nonempty sets such that the cardinality of $X_{\alpha} \neq 1$ or 2 for all $\alpha \in \Gamma$. Then $\mathscr{R}(X)$ coordinatizes the horizontal sum of the Boolean lattices $\mathscr{P}\left(X_{\alpha}\right), \alpha \in \Gamma$. The restriction that the cardinality of $X_{\alpha} \neq 1$ or $2, \alpha \in \Gamma$, is only included so that 3.2 is satisfied. As far as this example is concerned the cardinality restriction can be removed.

Some final comments are in order. In Gudder and Schelp (1970), orthomodular posets are coordinatized by OM-partial Baer *-semigroups so that it is only natural to wonder if and when $\mathscr{R}(X),\left\{X_{\alpha}\right\}_{\alpha e \Gamma}$ as in 2.1, satisfies condition OM. The "if" is settled by Example 4.2, since that $\mathscr{R}(X)$ is not OM even though $P^{\prime}(\mathscr{R}(C))$ is orthomodular. Also $P^{\prime}(\Theta(X))$ may very well be a non-orthomodular 
orthocomplemented poset for such a family $\left\{X_{\alpha}\right\}_{\alpha \in \Gamma}$. Thus it would be interesting to know necessary and sufficient conditions of the family $\left\{X_{\alpha}\right\}_{\alpha \in \Gamma}$ satisfying 2.1 such that $\Theta(X)$ is an OM-partial Baer *-semigroup and also conditions such thats $P^{\prime}(\mathscr{R}(X))$ is orthomodular. Finally since the coordinatization in 3.11 is for the somewhat restricted orthomodular posets satisfying Convention 3.1, it would be nice to determine the most general setting in which this theorem holds.

\section{References}

R. P. Dilworth (1940), 'On complemented lattices', Tohoku Math. Journal 47, 18-23.

D. J. Foulis (1960), Baer *-semigroups.', Proc. Amer. Math. Soc. 11, 468-654.

D. J. Foulis, Lectures notes on orthomodular lattices (Unpublished, University of Massachusetts Amherst. Massachusetts).

R. J. Greechie (1968), 'On the structure of orthomodular lattices satisfying the chain condition', J. Combinatorial Theory 5, 210-218.

R. J. Greechie (1971), 'Orthomodular lattices admitting no states', J. Combinatorial Theory (2) $5,119-132$.

S. P. Gudder and R. H. Schelp (1970), 'Coordinatiztion of orthocomplemented and orthomodular posets', Proc. Amer. Math. Soc. (2) 25, 229-237.

M. F. Janowitz (1963), Quantifiers on quasi-orthomodular lattices (Unpublished doctoral dissertation, Wayne State University, Detroit, Michigan, 1963).

Memphis State University

Memphis, Tennessee 38111

U.S.A. 